\title{
Rhomboid Flap vs. Keystone Perforator Island Flap (KPIF) in the Treatment of Pilonidal Sinus Disease: Comparison of Short-Term Results
}

\author{
Marius D. Roatis ${ }^{1}$, Alexandru V. Georgescu ${ }^{2}$ \\ ${ }^{1}$ Emergency County Hospital, Department of Plastic Surgery, Satu Mare, Romania \\ ${ }^{2}$ Plastic Surgery Reconstructive Microsurgery Clinic, University of Medicine and Pharmacy, Rehabilitation Hospital, Cluj-Napoca, \\ Romania \\ Email:dr_mariusdinu@yahoo.com
}

How to cite this paper: Roatis, M.D. and Georgescu, A.V. (2020) Rhomboid Flap vs. Keystone Perforator Island Flap (KPIF) in the Treatment of Pilonidal Sinus Disease: Comparison of Short-Term Results. International Journal of Clinical Medicine, 11, 454-464.

https://doi.org/10.4236/ijcm.2020.117038

Received: June 4, 2020

Accepted: July 24, 2020

Published: July 27, 2020

Copyright $\odot 2020$ by author(s) and Scientific Research Publishing Inc. This work is licensed under the Creative Commons Attribution International License (CC BY 4.0).

http://creativecommons.org/licenses/by/4.0/

\begin{abstract}
Background: Pilonidal disease is a chronic inflammatory disease of the sacrococcygeal region that mainly affects young people. Its incidence is 26 cases per 100,000 persons. Although many techniques have been described, there is no consensus on the treatment of pilonidal sinus disease (PSD). Materials and Methods: This study included 30 patients with PSD who were treated between May 2014 and September 2017. All cases underwent excision and flap reconstruction. The operative time, postoperative complications, the length of hospital stay, painless sitting and walking time, patient satisfaction and recurrence were evaluated prospectively. Results: The results of this prospective, randomized and comparative study are based on experience of a single surgical centre. All patients were followed up 18 months after discharge from the hospital. There is a difference in surgery durations (minutes) between the two groups (33.86 $\pm 2.89 \mathrm{~min}$. in "keystone" flap vs. $41.26 \pm 4.19$ in the "rhombic" flap group) $(\mathrm{p}=0.001)$. There were no significant differences in the length of hospital stay, painless sitting and walking time or patient satisfaction. The total complication rate was $66.6 \%$ after rhomboid flap compared with $6.6 \%$ after keystone flap. There was no flap necrosis. Conclusions: Both of these methods have shown to be successful in treatment of PSD. The KPIF is associated with the advantages of very simple design, abundant blood supply from the perforator vessels and lower rate of complication.
\end{abstract}

\section{Keywords}

Pilonidal Sinus Disease, Keystone Perforator Flap, Rhomboid Flap, Surgical Treatment 


\section{Introduction}

Pilonidal sinus disease is a common and acquired entity of young adults and has an estimated incidence of $26 / 100,000$ in the general population [1] [2]. It is seen more commonly in men than in women (male:female ratio 3 - 4:1) [3]. It usually affects the skin overlying the natal cleft at the sacrococcygeal area [4] and was first described by Anderson in 1847 [5]. Several short- and long-term outcomes have been published comparing different methods of treatments in PSD. Most of these studies compare Limberg rhomboid flap with other surgical or non-surgical techniques. There are no studies within the literature comparing rhomboid flap versus keystone perforator island flap (KPIF) although KPIF has been widely used in clinical practice since it was introduced. The aim of the present study was to compare the short-term results of the management of sacrococcygeal PSD with the keystone flap vs. the rhomboid flap (Limberg or Dufourmentel) in a randomized controlled trial as regards recurrence and complications rates, operative time, duration of hospital stay, time to walk without pain, time to sitting on toilet without pain, duration of the incapacity to work and cosmetic satisfaction of the patients.

\section{Materials and Methods}

\subsection{Patients}

Between May 2014 and September 2017, we included a series of 30 patients, prospectively and consecutively, which randomly divided into two groups. Fifteen patients (group 1) undergone elliptical excision and keystone perforator island flap (KPIF) and others 15 (group 2) undergone a rhomboid excision and the rhomboid flap to cover loss of substances after pilonidal cyst excision. The nature of surgical procedures was explained to the patients and informed consent had been obtained. Both surgical procedures were performed by the same surgeons. The surgeries were performed under spinal anesthesia and the patients were placed in the prone jack-knife position. All patients received a single intravenous dose of $2 \mathrm{~g}$ Sulcef (Cefoperazone sodium $500 \mathrm{mg}+$ Sulbactam sodium $500 \mathrm{mg}$, Novartis India Ltd.) with $30 \mathrm{~min}$. before surgery. Hair of the sacral and gluteal regions was shaved a few days preoperatively, and rectal cleansing with enemas was performed the night before the operations. The buttocks were separated with adhesive tape to allow wide exposure of the operative field. In the case of the keystone perforator island flap (KPIF), the parasacral perforators were identified and marked on the skin, using an acoustic Doppler technique, for safety only, although this is not absolutely necessary because the integument covering this region has plenty of perforators and vascular connections from lumbar and gluteal arteries. The skin was prepared with $10 \%$ povidone-iodine solution. In both techniques, methylene blue was injected in the pilonidal sinuses to guide the excision [6]. Dissection and hemostasis were performed by using electrocautery. 


\subsection{Randomization}

Patients were randomly assigned to undergo surgery with either the rhomboid flap or the keystone perforator island flap. Randomization was performed by using a sealed envelope that contained a paper with "Rhomboid flap" or "Keystone flap" which were opened before surgery.

\section{Surgical Technique}

\subsection{Keystone Perforator Island Flap (KPIF) (Figure 1) (Figure 2)}

The keystone perforator island flap (KPIF) was described by Behan in 2003 as a curvilinear trapezoidal shaped flap, representing the architectural shape of the keystone in Roman arches, relying on randomly fasciocutaneous or musculocutaneous perforators. It representing two opposing V-Y flaps joined together, with a flap width at a 1:1 ratio to an elliptical defect [7] [8]. Its length is determined by the size of the wound. An elliptical incision, including the pilonidal sinus, was made and a keystone flap was designed adjacent to the wound. The KPIF was incised along its sides without leaving a skin bridge intact and dissected down to the deep fasciae which was incised for further advancement, without or with minimal undermining to preserve the integrity of the vascular perforators [9]. Then, the flap padle was elevated and advanced to cover the defect. When we suture the flap on the defect-side it is very important to avoid dead space formation. Wound closure is achieved in a single layer using 1 Dafilon (B|Braun, Aesculap). The donor site was closed primarily. If the flap exceeds the 1: 1 ratio then more perforators can be incorporated into its structure thus creating a multiperforator advancement flap [8].

A
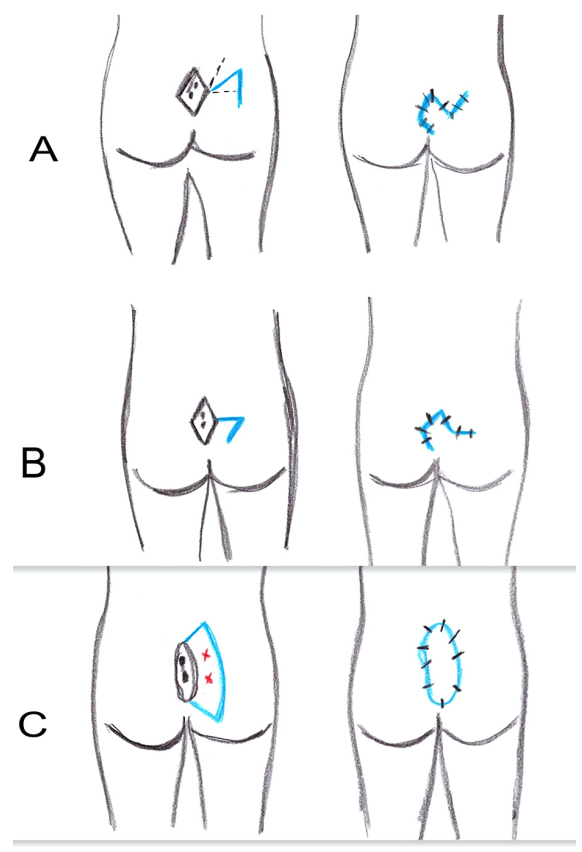

Figure 1. Diagram of surgical procedure (A) Dufourmentel flap (rhomboid); (B) Limberg flap (rhomboid); (C) Keystone perforator island flap. 

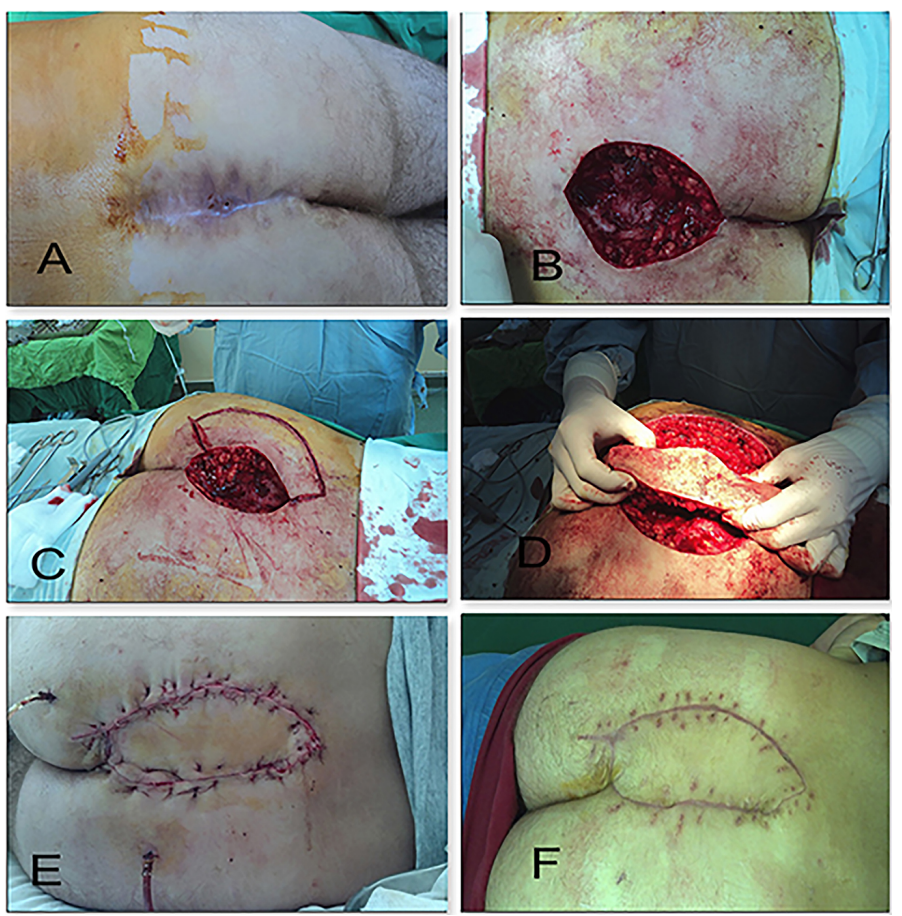

Figure 2. Keystone perforator island flap-clinical aspect (A) pilonidal sinus disease in sacrococcygeal area; (B) post-excisional defect; (C) the design of the flap; (D) the island-form and advancement of the flap; (E) postoperative clinical photography immediately after the flap inset; (F) aspect at 2 months postoperative.

\subsection{Rhomboid Flap (Figure 1) (Figure 3)}

Rhomboid flap was first described by Alexander Limberg in 1946 as a transposition flap with a random blood supply and it was modified by Dufourmentel in 1962 [10] [11]. In the Limberg flap, all angles are $60^{\circ}$ and $120^{\circ}$ and all sides are equal. The Dufourmentel flap has a safer blood supply than the Limberg flap because its base is wider [12]. The pilonidal sinus was included in a rhombic form and the flap was marked on the skin. Afterwards, the lesion was excised down to the presacral fascia under the guidance of methylene blue, and the fasciocutaneous flap was transposed medially to the defect [6]. A 2-layer closure was performed. The subcutaneous layers were approximated with 2 - 0 Vicryl (polyglactin 910, Ethicone, Johnson \& Johnson) interrupted sutures that fix the undersurface of the flap to the presacral fascia and avoid tension and obliterate the dead space. The skin was closed with 2 - 0 Dafilon (B|Braun, Aesculap) interrupted sutures [13]. In both procedures, the suction drain was placed beneath the flap through 1 or 2 separate stab incision.

\subsection{Postoperative Follow-Up}

All patients were followed-up in the outpatient clinic weekly in the first month following discharge from the hospital for wound assessment and then every 3 months to one year. The skin sutures were removed after 14 postoperative days. The suction drain was removed when the effluent was less than $20 \mathrm{ml}$ per day. 


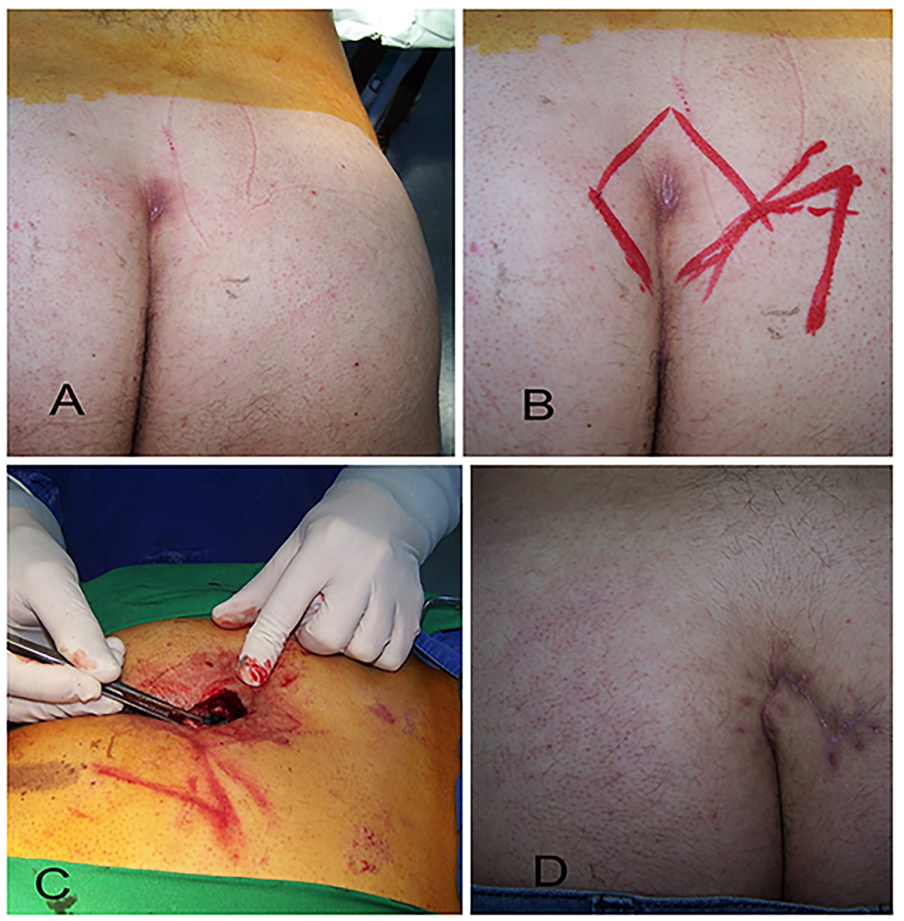

Figure 3. Rhomboid flap-clinical aspect (A) pilonidal sinus disease; (B), (C) Dufourmentel (rhomboid) flap; (D) Final result with stable coverage six weeks after surgery.

\subsection{Statistical Analyses}

All statistical analyses were performed using IBM SPSS Statistics for Mac. Student's t-test was used to compare continuous variables (quantitative data) between groups and Chi-squared or Fisher's exact test was used for categorical variables (qualitative data). The results are presented as mean plus or minus standard deviation for quantitative data or proportion, as appropriate. A two-sided $\mathrm{p}$ values of $<0.05$ were considered to indicate statistical significance.

\section{Results}

Of the 32 patients enrolled initially, 2 were lost to follow-up within 4 months of the procedure and were not included in the study. The remaining 30 patients: 24 males (80\%) and 6 females (20\%) were included in this analysis.

The average age of the included patients was 35.4 years (range: 20 - 50 years) in the "keystone" group and 27 years (range: $17-48$ years) in the "rhombic" group.

Operation time is important for the effectiveness of a surgical technique. It was defined as the time from the start of skin incision to the end of the last stitch. In terms of operative time, there was a slightly difference between the two groups: $33.86 \pm 2.89 \mathrm{~min}$. in the "keystone" flap group vs. $41.26 \pm 4.19 \mathrm{~min}$. in the "rhombic" flap group $(\mathrm{p}=0.001)$ (Table 1$)$.

The seroma, which is defined as the formation of non-infected serous fluid collection beneath the flap, has a formation rate significantly higher in the 
rhomboid group ( 3 patients-20\%) than in the keystone group (0 patients) (Table $1)$.

No patient in the keystone group had wound infection, but this complication occurred in 2 patients $(13.3 \%)$ in the rhomboid flap group $(\mathrm{p}=0.526)$. These two patients were treated with oral antibiotic therapy for 7 days (Table 1).

The rates of wound dehiscence in group 1 was $6.66 \%$ (1 patient and in group 2 were $20 \%$ ( 3 patients). All of these patients healed by second intention without requiring additional surgical procedures (Table 1).

The duration of hospital stay is defined as the number of days from the operation day until the day of discharge.

The average length of hospital stay for the "rhombic" group was longer than "keystone" group ( $4 \pm 1.98$ days versus $2.33 \pm 0.487$ days, $\mathrm{p}=0.003)$, suture disruption and infections are likely to increase the duration of hospitalization (Table 2).

There were no statistically significant differences between the two groups regarding time to walk pain-free (in days): $9.06 \pm 1.48$ in group 1 and $9.60 \pm 1.45$

Table 1. The distribution of complications within the 2 groups.

\begin{tabular}{ccccc}
\hline Complication & $\begin{array}{c}\text { Total } \\
(\mathrm{n}=30)\end{array}$ & $\begin{array}{c}\text { Group 1 } \\
(\mathrm{n}=15)\end{array}$ & $\begin{array}{c}\text { Group 2 } \\
(\mathrm{n}=15)\end{array}$ & P value \\
\hline Minor complications & $9(30)$ & $1(6.66)$ & $8(53.33)$ & \\
Seroma & $3(10)$ & 0 & $3(20)$ & 0.224 \\
Wound dehiscence & $4(13.33)$ & $1(6.66)$ & $3(20)$ & 0.598 \\
Wound infection & $2(6.66)$ & 0 & $2(13.33)$ & 0.483 \\
Major complications & & - & & \\
Total or partial flap necrosis & - & 0 & $2(13.33)$ & 0.483 \\
Recurrence & $2(6.66)$ & &
\end{tabular}

Values are presented as number and (\%).

Table 2. Patients VAS score, time to walking pain-free and time to sitting on toilet without pain, incapacity for work (days) and hospital stay (days).

\begin{tabular}{cccc}
\hline Parameters & $\begin{array}{c}\text { Group 1 } \\
(\mathrm{n}=15)\end{array}$ & $\begin{array}{c}\text { Group 2 } \\
(\mathrm{n}=15)\end{array}$ & P value \\
\hline VAS score & $7.13 \pm 1.18^{*}$ & $7.0 \pm 1.0^{*}$ & 0.742 \\
Time to walking pain-free & $9.06 \pm 1.48^{*}$ & $9.60 \pm 1.45^{*}$ & 0.329 \\
Time to sitting on toilet without pain & $10.26 \pm 1.03^{*}$ & $11.8 \pm 2.17^{*}$ & 0.032 \\
Incapacity for work (days) & $21.93 \pm 1.94^{*}$ & $23.4 \pm 3.83^{*}$ & 0.017 \\
Hospital stay (days) & $2.33 \pm 0.487^{*}$ & $4 \pm 1.98^{*}$ & 0.003 \\
\hline
\end{tabular}

*mean. 
in group $2,(\mathrm{p}=0.329)$ and slight difference as for time to sitting on toilet without pain (in days), $10.26 \pm 1.03$ and $11.8 \pm 2.17$, respectively ( $\mathrm{p}=0.032$ ) (Table 2).

Recurrent disease occurred in 2 patients (13.3\%) who undergone rhomboid flap (group 2) in the 11th and 14th postoperative months. No patients had recurrence in the "keystone" group (group 1). All cases were followed up for 18 months.

Time away from work was defined as the date on which patient returned to employment from the date of surgery. The postoperative duration of incapacity for work (days) was longer in the "rhomboid" group than in the "keystone" group $(23.4 \pm 3.83$ days vs. $21.93 \pm 1.94$ days $)(\mathrm{p}=0.017)($ Table 2$)$.

The visual analogue scale (VAS) score from 1 - 10 (0 for "very bad" to 10 for "very good") was used in order to assess the cosmetic satisfaction of the patients.

The cosmetic satisfaction was determined at the end of the third postoperative month by asking patients to describe their satisfaction with the operation scar due to pilonidal sinus disease.

The VAS score for satisfaction with the cosmetic appearance of the scars in "keystone" group was $7.13 \pm 1.18$, whereas it was $7.0 \pm 1.0$ in the "rhomboid" group $(\mathrm{p}=0.742)($ Table 2$)$.

No patients developed flap necrosis in the two groups.

There were no donor site complications.

\section{Discussion}

The etiopathogenesis of SPD is still under debate, based on two principal theories: "congenital" theory (the absence of coalescence of the primitive ectoderm) and "acquired" theory (the hair follicle has the primordial role) [14].

The ideal surgical procedure for sacrococcygeal PSD should be simple and quick, should not require a long hospital stay, should have low recurrences rate and postoperative complications, low cost and high patient satisfaction [15] [16].

Many surgical procedures for SPD have been developed, but none of them fulfill all of these features. All of surgical techniques have advantages and disadvantages.

Excision of the cyst is not technically difficult but how to cover the surgical defect after excision of sacrococcygeal pilonidal sinus may be sometimes a dilemma.

Of all the surgical procedures, the local flaps fill the defect, avoid a midline scar, provide tension free closure and can flatten the natal cleft and thereby offer advantages over direct closure techniques [17].

The rhomboid flap is one of the most common surgical techniques used in PSD forasmuch is easy to design, raise and inset.

It has gained popularity because it is associated with low complication and recurrence rates [15].

However, in the rhomboid flap large tissue should be displacements and the 
flap is usually a random pattern, which limits the size of flap that can be raised. As it looks like Arpaci et al. the lower pole of the rhomboid flap can be a weak point in terms of recurrence [18]. On the contrary, the keystone flap does not require extensive mobilization and it does not require fixation at the sacral fascia and thus the operative time is lower than then when we use rhomboid flap (33.86 \pm 2.89 min vs. $41.26 \pm 4.19$ in our study).

In KPIF the wound tension is effectively reduced and redistributed and thus the healing process is facilitated [19]. The KPIF has been widely used in clinical practice since it was first introduced for the reconstruction of defects located on the head and neck, trunk, and extremities. The KPIF can be divided into four subtypes: type I the classical flap, in which the flap is superficially disected of deep fascia; type IIA requires division of the deep fascia; type IIB that cannot be closed without a skin graft due to the tension, type III, which consists of two KDPIFs; and type IV that is a rotational keystone flap [7].

KPIF is a multiperforator advancement flap based on random fasciocutaneous or musculocutaneous perforator but in the sacral area, these may be parasacral perforators arise from the lateral sacral artery or the internal pudendal artery as described Koshima and Ahmadzadeh [20] [21].

The most important factors to consider when evaluating the results of the surgical treatment are: early complications and delayed relaps [6].

Ersoy et al. reported wound infection rate after the Limberg flap (8\%) [22]. Altintoprak et al. had 3.3\% (11 patients) rate of infection on 324 patients after Limberg flap procedure. In our study, no patients have wound infection after KPIF but infection rate in rhomboid flap was $13.3 \%$.

The main aim of the surgical treatment of pilonidal sinus disease remains prevention of recurrence.

A meta-analysis of more than 100 randomized controlled trials found that the recurrence rate at 5 years overall surgical therapies for pilonidal disease was 20.3\% [3] [4] [23].

Topgül et al. on 200 operated patients reported $2.5 \%$ recurrence rate for Limberg flap and Daphan et al. on 147 operated patients noted 4.8\% recurrence [24].

We had no recurrence with keystone flap but in rhomboid group we had 2 patients with recurrence at 18 months.

The hospital stay is also an important criterion in determining the success of a surgical technique.

Mentes et al., in their study, on 353 patients operated, reported $4.51 \pm 2.85$ days of hospital stay, Topgül et al., 3.1 days on 200 patients, Erdem et al., $3.5 \pm$ 1.16 days on 40 patients and Eryilmaz et al., 3 days on 63 patients [24].

In our study, we operated 30 patients, and the mean length hospital stay was 4 \pm 1.98 days for "rhomboid" group and $2.33 \pm 0.487$ days for "keystone" group.

In contrast to the direct suture, in the case of cutaneous flaps, the scars are larger and, therefore, its can be sometimes unpleasant for the patient. The cosmetic appearance of the scars may be more important for women than men. 
Eryilmaz et al., reported that as many as $40 \%$ of patients were not pleased with the appearance of Limberg flap scars [17] [25]. In our study, the cosmetic appearance of the scars at the operation site was accepted by the majority of the thirty patients. No significant difference was found between patients treated with the keystone flap technique and those treated with the rhomboid flap technique. This was an issue especially for female patients, that expressed their dissatisfaction with the cosmetic outcome for both procedures. All these results encourage to use keystone flap technique in the surgical management of PSD.

\section{Conclusions}

The keystone and rhomboid flaps are useful techniques to close wounds after pilonidal cyst and both of them achieve off-midline closure and flattening the natal cleft.

The KPIF, unlike rhombic flap techniques, avoids extensive mobilization of tissue and maintains good blood supply with minimal tension. Where a larger excision is required, we can use the modified KPIF.

Although, both of these methods have shown to be successful, the keystone flap technique seems to be a safer method. We successfully managed PSD with excision and KPIF reconstruction, without recurrence and minimal postoperative complications. In conclusion, we believe that KPIF is a good alternative choice in the management of PSD because its elevation is easy, fast, and safe.

\section{Limitations and Future Work}

The mean follow-up time for patients in this study was 18 months and this comprised 30 patients. This period is short for the evaluation of recurrences. A larger patient population and a longer follow-up time are required.

\section{Consent}

All included patients provided their written informed consent, and the study's protocol was approved by the research ethics committee of Emergency County Hospital Satu Mare.

\section{Conflicts of Interest}

The authors declare no conflicts of interest regarding the publication of this paper.

\section{References}

[1] Saydam, M., Ozturk, B., Sinan, H., Balta, A. Z., Demir, P., Ozer, M.T. and Demirbas, S. (2015) Comparison of Modified Limberg Flap Transposition and Lateral Advancement Flap Transposition with Burow's Triangle in the Treatment of Pilonidal Sinus Disease. The American Journal of Surgery, 210, 772-777. https://doi.org/10.1016/j.amjsurg.2015.03.031

[2] Rashidian, N., Vahedian-Ardakani, J., Baghai-Wadji, M., Keramati, M.R., Saraee, A., Ansari, K. and Adman, A.A. (2014) How to Repair the Surgical Defect after Ex- 
cision of Sacrococcygeal Pilonidal Sinus: A Dilemma. Journal of Wound Care, 23, 630-633. https://doi.org/10.12968/jowc.2014.23.12.630

[3] Horwood, J., Hanratty, D., Chandran, P. and Billings, P. (2012) Primary Closure or Rhomboid Excision and Limberg Flap for the Management of Primary Sacrococcygeal Pilonidal Disease? A Meta-Analysis of Randomized Controlled Trials. Colorectal Disease, 14, 143-151. https://doi.org/10.1111/j.1463-1318.2010.02473.x

[4] Eastment, J. and Slater, K. (2020) Outcomes of Minimally Invasive Endoscopic Pilonidal Sinus Surgery. Asian Journal of Endoscopic Surgery, 13, 324-328. https://doi.org/10.1111/ases.12748

[5] Bali, İ., Aziret, M., Sözen, S., Emir, S., Erdem, H., Çetinkünar, S. and İrkörücü, O. (2015) Effectiveness of Limberg andkarydakis Flap in Recurrent Pilonidal Sinus Disease. Clinics (Sao Paulo), 70, 350-355. https://doi.org/10.6061/clinics/2015(05)08

[6] Ates, M., Dirican, A., Sarac, M., Aslan, A. and Colak, C. (2011) Short and Long-Term Results of the Karydakis Flap versus the Limberg Flap for Treating Pilonidal Sinus Disease: A Prospective Randomized Study. The American Journal of Surgery, 202, 568-573. https://doi.org/10.1016/j.amjsurg.2010.10.021

[7] Behan, F.C. (2003) The Keystone Design Perforator Island Flap in Reconstructive Surgery. ANZ Journal of Surgery, 73, 112-120. https://doi.org/10.1046/j.1445-2197.2003.02638.x

[8] Mohan, A.T., Rammos, C.K., Akhavan, A.A., Martinez, J., Wu, P.S., Moran, S.L., et al. (2016) Evolving Concepts of Keystone Perforator Island Flaps (KPIF): Principles of Perforator Anatomy, Design Modifications, and Extended Clinical Applications Plastic and Reconstructive Surgery, 137, 1909-1920. https://doi.org/10.1097/PRS.0000000000002228

[9] Wu, M.L., Sun, M.Y., Dai, H.Y., Xu, J.G., Wang, X.W., Guo, R., Wang, Y.C. and Xue, C.Y. (2019) The Application of Keystone Flap Combined with Vacuum-Assisted Closure in the Repair of Sacrococcygeal Skin Defect after Tumor Resection. Journal of Surgical Oncology, 119, 974-978. https://doi.org/10.1002/jso.25397

[10] Federico, T., Mario, C., Stefano, S., Claudia, F. and Luigi, V. (2012) A Modified Rhomboid Flap: The "Diamond Flap". Dermatologic Surgery, 38, 1851-1855. https://doi.org/10.1111/j.1524-4725.2012.02557.x

[11] Turan, T., Kuran, I., Ozcan, H. and Baş, L. (1999) Geometric Limit of Multiple Local Limberg Flaps: A Flap Design. Plastic and Reconstructive Surgery, 104, 1675-1678. https://doi.org/10.1097/00006534-199911000-00010

[12] Lister, G.D. and Gibson, T. (1972) Closure of Rhomboid Skin Defects: The Flaps of Limberg and Dufourmentel. British Journal of Plastic Surgery, 25, 300-314. https://doi.org/10.1016/S0007-1226(72)80067-5

[13] Mahdy, T. (2008) Surgical Treatment of the Pilonidal Disease: Primary Closure or Flap Reconstruction after Excision. Diseases of the Colon \& Rectum, 51, 1816-1822. https://doi.org/10.1007/s10350-008-9436-8

[14] De Parades, V., Bouchard, D., Janier, M. and Berger, A. (2013) Pilonidal Sinus Disease. Journal of Visceral Surgery, 150, 237-247. https://doi.org/10.1016/j.jviscsurg.2013.05.006

[15] Sahebally, S.M., Mcmahon, G., Walsh, S.R. and Burke, J.P. (2018) PWE-018 Classical Limberg versus Karydakis Flaps for Pilonidal Disease-A Meta-Analysis of Randomised Controlled Trials. Gut 2017, 66, A134. https://doi.org/10.1136/gutjnl-2017-314472.263

[16] Yildiz, T., Ilce, Z. and Kücük, A. (2014) Modified Limberg Flap Technique in the 
Treatment of Pilonidal Sinus Disease in Teenagers. Journal of Pediatric Surgery, 49, 1610-1613. https://doi.org/10.1016/j.jpedsurg.2014.06.011

[17] Venus, M. and Titley, O. (2012) Outcomes in the Repair of Pilonidal Sinus Disease Excision Wounds Using a Parasacral Perforator Flap. The Annals of the Royal College of Surgeons of England, 94, 12-16. https://doi.org/10.1308/003588412X13171221499748

[18] Arpaci, E., Altun, S., Orhan, E., Eyuboglu, A. and Ertas, N.M. (2018) A New Oval Advancement Flap Design for Reconstruction of Pilonidal Sinus Defect. World Journal of Surgery, 42, 3568-3574. https://doi.org/10.1007/s00268-018-4648-0

[19] Yoon, C.S., Kim, H.B., Kim, Y.K., Kim, H. and Kim, K.N. (2019) Keystone-Design Perforator Island Flaps for the Management of Complicated Epidermoid Cysts on the Back. Scientific Reports, 9, Article No. 14699.

https://doi.org/10.1038/s41598-019-51289-4

[20] Koshima, I., Moriguchi, T., Soeda, S., et al. (1993) The Gluteal Perforator-Based Flap for Repair of Sacral Pressure Sores. Plastic Reconstructive Surgery, 91, 678-683. https://doi.org/10.1097/00006534-199304000-00017

[21] Ahmadzadeh, R., Bergeron, L., Tang, M., et al. (2007) The Superior and Inferior Gluteal Artery Perforator Flaps. Plastic Reconstructive Surgery, 120, 1551-1556. https://doi.org/10.1097/01.prs.0000282098.61498.ee

[22] Ersoy, E., Devay, A.O., Aktimur, R., et al. (2009) Comparison of the Short-Term Results after Limberg and Karydakis Procedures for Pilonidal Disease: Randomized Prospective Analysis of 100 Patients. Colorectal Disease, 11, 705-710. https://doi.org/10.1111/j.1463-1318.2008.01646.x

[23] Stauffer, V.K., Luedi, M.M., Kauf, P., et al. (2018) Common Surgical Procedures in Pilonidal Sinus Disease: A Meta-Analysis, Merged Data Analysis, and Comprehensive Study on Recurrence. Scientific Reports, 8, Article No. 3058. https://doi.org/10.1038/s41598-018-20143-4

[24] Mentes, O., Bagci, M., Bilgin, T., Ozgul, O. and Ozdemir, M. (2007) Limberg Flap Procedure for Pilonidal Sinus Disease: Results of 353 Patients. Langenbeck's Archives of Surgery, 393, 185-189. https://doi.org/10.1007/s00423-007-0227-9

[25] Eryilmaz, R., Sahin, M., Alimoglu, O., et al. (2003) Surgical Treatment of Sacrococcygeal Pilonidal Sinus with the Limberg Transposition Flap. Surgery, 134, 745-749. https://doi.org/10.1016/S0039-6060(03)00163-6 